\title{
Existence and uniqueness of solutions for a mixed $p$-Laplace boundary value problem involving fractional derivatives
}

\author{
Shuqi Wang ${ }^{1}$ and Zhanbing Bai ${ }^{1 *}$ (D)
}

\section{"Correspondence:}

zhanbingbai@163.com

'College of Mathematics and

System Science, Shandong

University of Science and

Technology, Qingdao 266590,

P.R. China

\section{Springer}

\begin{abstract}
In this article, the existence and uniqueness of solutions for a multi-point fractional boundary value problem involving two different left and right fractional derivatives with $p$-Laplace operator is studied. A novel approach is used to acquire the desired results, and the core of the method is Banach contraction mapping principle. Finally, an example is given to verify the results.
\end{abstract}

MSC: 34A08; 34B15; 35J05

Keywords: Fractional derivatives; $p$-Laplace operator; Existence and uniqueness

\section{Introduction}

In recent years, fractional-order calculus theory has been widely used in mathematics, science, engineering, etc. As a result, the studies of such equation have gained considerable popularity, see [1-8]. Also, fractional-order mixed differential or integral equation involving different fractional derivatives, such as conformable fractional, Riemann-Liouville, and Caputo, has got a lot of interest, and even fractional-order differential or integral equations with $p$-Laplace operator have been extensively discussed by more and more researchers [9-14].

In [11], a mixed fractional $p$-Laplace boundary value problem was studied by Liu et al.

$$
\left\{\begin{array}{l}
D_{0+}^{\alpha}\left(\varphi_{p}\left({ }^{c} D_{0+}^{\beta} u(t)\right)\right)=f\left(t, u(t),{ }^{c} D_{0+}^{\beta} u(t)\right), \\
{ }^{c} D_{0+}^{\beta} u(0)=u^{\prime}(0)=0, \\
u(1)=r_{1} u(\eta), \quad{ }^{c} D_{0+}^{\beta} u(1)=r_{2}{ }^{c} D_{0+}^{\beta} u(\xi),
\end{array}\right.
$$

where $\varphi_{p}(t)=|t|^{p-2} \cdot t, p>1,1<\alpha, \beta \leq 2, r_{1}, r_{2} \geq 0, D_{0+}^{\alpha}$ is Riemann-Liouville fractional derivative, ${ }^{c} D_{0+}^{\beta}$ is Caputo fractional derivative, and $f:[0,1] \times[0,+\infty) \times(-\infty, 0] \rightarrow$ $[0,+\infty)$ is continuous. The minimum upper solution and the maximum lower solution of the above boundary value problem were given by applying the lower and upper solutions method.

(c) The Author(s) 2020. This article is licensed under a Creative Commons Attribution 4.0 International License, which permits use, sharing, adaptation, distribution and reproduction in any medium or format, as long as you give appropriate credit to the original author(s) and the source, provide a link to the Creative Commons licence, and indicate if changes were made. The images or other third party material in this article are included in the article's Creative Commons licence, unless indicated otherwise in a credit line to the material. If material is not included in the article's Creative Commons licence and your intended use is not permitted by statutory regulation or exceeds the permitted use, you will need to obtain permission directly from the copyright holder. To view a copy of this licence, visit http://creativecommons.org/licenses/by/4.0/. 
In [2], Bai investigated the uniqueness and existence of solutions of the following fractional-order differential equation:

$$
\left\{\begin{array}{l}
\left(\varphi_{p}\left(D_{0+}^{\alpha} u(t)\right)\right)^{\prime}+f(t, u(t))=0, \\
u(0)=D_{0+}^{\alpha} u(0)=0, \quad{ }^{c} D_{0+}^{\beta} u(1)={ }^{c} D_{0+}^{\beta} u(1)=0,
\end{array}\right.
$$

where $D_{0+}^{\alpha}$ is Riemann-Liouville fractional derivative, ${ }^{c} D_{0_{+}}^{\beta}$ is Caputo fractional derivative, $0<\beta \leq 1,2<\alpha \leq 2+\beta, p>1$, and $f:[a, b] \times \mathbb{R} \rightarrow \mathbb{R}$ is continuous. The uniqueness result of a solution and the existence of specific solutions to problem were showed by applying Guo-Krasnoselskii's fixed point theorem.

In [4], Dang et al. proposed a fresh approach to gain the existence and uniqueness of solutions of a fourth-order two-point nonlinear differential equation

$$
\begin{aligned}
& u^{(4)}(x)=f\left(x, u(x), u^{\prime \prime}(x)\right), \\
& u(0)=u(1)=u^{\prime \prime}(0)=u^{\prime \prime}(1)=0,
\end{aligned}
$$

where $f:[0,1] \times \mathbb{R}^{2} \rightarrow \mathbb{R}$ is continuous. In [3], Bai et al. considered a class of fourthorder nonlinear differential equation with $p$-Laplace operator, and the boundary conditions change from two points to multiple points compared to the above problem. In both papers, the main results were given by applying the Banach contraction mapping principle.

As far as we know, nobody used the method which was put forward by Dang [6] and Bai [3] to prove the existence and uniqueness of solutions of a nonlinear multi-point fractional-order $p$-Laplace boundary value problem, which has at least two different kinds of fractional derivatives. Inspired by the above-mentioned articles, the following mixed boundary value problem is studied in this work:

$$
\left\{\begin{array}{l}
{ }^{c} D_{1_{-}}^{\gamma}\left(\varphi_{p}\left(D_{0_{+}}^{\delta} y(x)\right)\right)=g\left(x, y(x), D_{0+}^{\delta} y(x)\right), \\
y(0)=0, \quad y(1)=r_{1} y(\mu), \\
D_{0+}^{\delta} y(1)=0, \quad \varphi_{p}\left(D_{0+}^{\delta} y(0)\right)=r_{2} \varphi_{p}\left(D_{0+}^{\delta} y(\eta)\right),
\end{array}\right.
$$

where $1<\gamma, \delta \leq 2,0<\mu, \eta<1,0 \leq r_{1}<\frac{1}{\mu^{\delta-1}}, 0 \leq r_{2}<\frac{1}{1-\eta}, \varphi_{p}(x)=|x|^{p-2} \cdot x, \frac{1}{p}+\frac{1}{q}=1$, $p, q>1, D_{0_{+}}^{\delta}$ is left Riemann-Liouville fractional derivative, ${ }^{c} D_{1-}^{\gamma}$ is right Caputo fractional derivative, and function $g \in C\left([0,1] \times \mathbb{R}^{2}, \mathbb{R}\right)$.

The rest of this work is organized as follows. In Sect. 2, some related definitions and necessary lemmas of fractional calculus theory are presented, which will be applied in the main results of this article. In Sect. 3, the existence and uniqueness of solutions of the mixed fractional-order $p$-Laplace differential equation are proved by applying the method which was put forward by Dang and Bai. In Sect. 4, a particular example is constructed to verify the main conclusions of the paper.

\section{Preliminaries}

This section introduces some related definitions and necessary lemmas of fractional calculus theory. 
Definition 2.1 For given $\gamma>0$ and the function $y:(0, \infty) \rightarrow \mathbb{R}$, define the left and right Riemann-Liouville fractional integrals respectively as follows:

$$
\begin{aligned}
& I_{0+}^{\gamma} y(x)=\frac{1}{\Gamma(\gamma)} \int_{0}^{x}(x-s)^{\gamma-1} y(s) d s, \\
& I_{1-}^{\gamma} y(x)=\frac{1}{\Gamma(\gamma)} \int_{x}^{1}(s-x)^{\gamma-1} y(s) d s .
\end{aligned}
$$

Definition 2.2 For given $\gamma>0, \gamma \in(n, n+1)$ and the function $y:(0, \infty) \rightarrow \mathbb{R}$, define the left Riemann-Liouville fractional derivative and the right Caputo fractional derivative respectively as follows:

$$
\begin{aligned}
& D_{0+}^{\gamma} y(x)=\frac{d^{n}}{d x^{n}} \frac{1}{\Gamma(n-\gamma)} \int_{0}^{x}(x-s)^{n-\gamma-1} y(s) d s, \\
& { }^{c} D_{1-}^{\gamma} y(x)=(-1)^{n} \frac{1}{\Gamma(n-\gamma)} \int_{x}^{1}(s-x)^{n-\gamma-1} y^{(n)}(s) d s .
\end{aligned}
$$

Let $E=C[0,1]$, whose norm $\|\cdot\|$ is the maximum norm. Given $\phi \in C[0,1]$ and the constants $r_{1}, r_{2} \in \mathbb{R}$, discuss the following fractional-order mixed $p$-Laplace boundary value problem:

$$
\left\{\begin{array}{l}
{ }^{c} D_{1-}^{\gamma}\left(\varphi_{p}\left(D_{0+}^{\delta} y(x)\right)\right)=\phi(x), \\
y(0)=0, \quad y(1)=r_{1} y(\mu), \\
D_{0+}^{\delta} y(1)=0, \quad \varphi_{p}\left(D_{0+}^{\delta} y(0)\right)=r_{2} \varphi_{p}\left(D_{0+}^{\delta} y(\eta)\right),
\end{array}\right.
$$

where $1<\gamma, \delta \leq 2,0<\mu, \eta<1,0 \leq r_{1}<\frac{1}{\mu^{\delta-1}}, 0 \leq r_{2}<\frac{1}{1-\eta}$.

Lemma 2.1 The unique solution of the fractional-order mixed p-Laplace boundary value problem (2.1) is equivalent to

$$
y(x)=\int_{0}^{1} G_{1}(x, \tau) \varphi_{q}\left(\int_{0}^{1} G_{2}(\tau, s) \phi(s) d s\right) d \tau
$$

where

$$
G_{1}(x, \tau)=\frac{1}{\Gamma(\delta)} \begin{cases}\Lambda_{1}\left[(1-\tau)^{\delta-1}-r_{1}(\mu-\tau)^{\delta-1}\right]-(x-\tau)^{\delta-1}, & 0<\tau \leq \min \{x, \mu\} ; \\ \Lambda_{1}\left[(1-\tau)^{\delta-1}-r_{1}(\mu-\tau)^{\delta-1}\right], & x \leq \tau \leq \mu ; \\ \Lambda_{1}(1-\tau)^{\delta-1}-(x-\tau)^{\delta-1}, & \mu \leq \tau \leq x ; \\ \Lambda_{1}(1-\tau)^{\delta-1}, & \max \{x, \mu\} \leq \tau<1,\end{cases}
$$

and

$$
G_{2}(\tau, s)=\frac{1}{\Gamma(\gamma)} \begin{cases}\Lambda_{2} s^{\gamma-1}, & 0<s \leq \min \{\tau, \eta\} \\ \Lambda_{2} s^{\gamma-1}-(s-\tau)^{\gamma-1}, & \tau \leq s \leq \eta \\ \Lambda_{2}\left[s^{\gamma-1}-r_{2}(s-\eta)^{\gamma-1}\right], & \eta \leq s \leq \tau ; \\ \Lambda_{2}\left[s^{\gamma-1}-r_{2}(s-\eta)^{\gamma-1}\right]-(s-\tau)^{\gamma-1}, & \max \{\tau, \eta\} \leq s<1\end{cases}
$$


with

$$
\Lambda_{1}=\frac{x^{\delta-1}}{1-r_{1} \mu^{\delta-1}}, \quad \Lambda_{2}=\frac{1-x}{1-r_{2}(1-\eta)} .
$$

Moreover, $G_{1}(x, \tau)>0, G_{2}(\tau, s)>0$ for $x, \tau, s \in(0,1)$.

Proof Let $-\varphi_{p}\left(D_{0+}^{\delta} y(x)\right)=k(x)$, then the mixed boundary value problem (2.1) changes to

$$
\left\{\begin{array}{l}
{ }^{c} D_{1-}^{\gamma} k(x)=-\phi(x), \\
k(1)=0, \quad k(0)=r_{2} k(\eta),
\end{array}\right.
$$

and

$$
\left\{\begin{array}{l}
D_{0+}^{\delta} y(x)=-\varphi_{q}(k(x)), \\
y(0)=0, \quad y(1)=r_{1} y(\mu) .
\end{array}\right.
$$

Reduce the equation ${ }^{c} D_{1-}^{\gamma} k(x)=-\phi(x)$ as an equivalent equation

$$
k(x)=-\frac{1}{\Gamma(\gamma)} \int_{x}^{1}(s-x)^{\gamma-1} \phi(s) d s+c_{1}+c_{2}(1-x) .
$$

Using the condition $k(1)=0$ yields $c_{1}=0$. Since $k(0)=r_{2} k(\eta)$, then

$$
k(0)=-\frac{1}{\Gamma(\gamma)} \int_{0}^{1} s^{\gamma-1} \phi(s) d s+c_{2}
$$

and

$$
k(\eta)=-\frac{1}{\Gamma(\gamma)} \int_{\eta}^{1}(s-\eta)^{\gamma-1} \phi(s) d s+c_{2}(1-\eta) .
$$

By calculation, we can get

$$
c_{2}=\frac{1}{\Gamma(\gamma)\left[1-r_{2}(1-\eta)\right]}\left(\int_{0}^{1} s^{\gamma-1} \phi(s) d s-\int_{\eta}^{1} r_{2}(s-\eta)^{\gamma-1} \phi(s) d s\right) .
$$

So,

$$
\begin{aligned}
k(x)= & -\frac{1}{\Gamma(\gamma)} \int_{x}^{1}(s-x)^{\gamma-1} \phi(s) d s \\
& +\frac{1-x}{\Gamma(\gamma)\left[1-r_{2}(1-\eta)\right]}\left(\int_{0}^{1} s^{\gamma-1} \phi(s) d s-\int_{\eta}^{1} r_{2}(s-\eta)^{\gamma-1} \phi(s) d s\right) \\
= & \int_{0}^{1} G_{2}(x, s) \phi(s) d s .
\end{aligned}
$$

Similarly, the solution of boundary value problem (2.4) is given by

$$
y(x)=\int_{0}^{1} G_{1}(x, s) \varphi_{q}(k(s)) d s .
$$

Consequently, boundary value problem (2.1) is equivalent to (2.2). 
From the monotonicity, for $x, \tau, s \in(0,1), G_{1}(x, \tau), G_{2}(\tau, s)>0$ are verified easily. The proof is completed.

Lemma 2.2 For given $\phi(s) \in C[0,1]$, set

$$
\begin{aligned}
& w(\tau)=\varphi_{q}\left(\int_{0}^{1} G_{2}(\tau, s) \phi(s) d s\right), \quad y(x)=\int_{0}^{1} G_{1}(x, \tau) w(\tau) d \tau, \\
& M_{1}=\max _{0 \leq x \leq 1} \int_{0}^{1} G_{1}(x, \tau) d \tau, \quad M_{2}=\max _{0 \leq \tau \leq 1} \int_{0}^{1} G_{2}(\tau, s) d s .
\end{aligned}
$$

Then

$$
\|w\| \leq M_{2}^{q-1}\|\phi\|^{q-1}, \quad\|y\| \leq M_{1} M_{2}^{q-1}\|\phi\|^{q-1} .
$$

Proof Since $\frac{1}{p}+\frac{1}{q}=1$ and $\varphi_{p}$ is increasing, then

$$
\begin{aligned}
w(\tau) & =\varphi_{q}\left(\int_{0}^{1} G_{2}(\tau, s) \phi(s) d s\right) \\
& \leq \varphi_{q}\left(\int_{0}^{1} G_{2}(\tau, s) d s\|\phi\|\right) \\
& \leq M_{2}^{q-1}\|\phi\|^{q-1} .
\end{aligned}
$$

So, $\|w\| \leq M_{2}^{q-1}\|\phi\|^{q-1}$. Similarly, $\|y\| \leq M_{1} M_{2}^{q-1}\|\phi\|^{q-1}$. The proof is completed.

Lemma 2.3 ([7, 13]) The following relations of $p$-Laplace operator hold:

(i) There are $1<p \leq 2,\left|k_{1}\right|,\left|k_{2}\right| \geq n>0$, and $k_{1} k_{2}>0$ such that

$$
\left|\varphi_{p}\left(k_{2}\right)-\varphi_{p}\left(k_{1}\right)\right| \leq(p-1) n^{p-2}\left|k_{2}-k_{1}\right|
$$

(ii) There are $p>2$ and $\left|k_{1}\right|,\left|k_{2}\right| \leq N$ such that

$$
\left|\varphi_{p}\left(k_{2}\right)-\varphi_{p}\left(k_{1}\right)\right| \leq(p-1) N^{p-2}\left|k_{2}-k_{1}\right|
$$

\section{Main results}

This section studies the existence and uniqueness of solutions for mixed fractional-order $p$-Laplace boundary value problem (1.1) by applying the Banach contraction mapping principle.

Given number $M>0$, denote

$$
D_{M}=\left\{(x, y, w)|0 \leq x \leq 1,| y\left|\leq M_{1} M_{2}^{q-1} M^{q-1},\right| w \mid \leq M_{2}^{q-1} M^{q-1}\right\}
$$

and by a closed ball $B[O, M]$ in the space of continuous functions $C[0,1]$.

Theorem 3.1 Assume that $1<p \leq 2$, and there exist some numbers $M, Q_{1}, Q_{2}>0$ such that the following conditions hold:

(H1) $|g(x, y, w)| \leq M$ for $(x, y, w) \in D_{M}$; 
(H2) $\left|g\left(x, y_{2}, w_{2}\right)-g\left(x, y_{1}, w_{1}\right)\right| \leq Q_{1}\left|y_{2}-y_{1}\right|+Q_{2}\left|w_{2}-w_{1}\right|$ for $\left(x, y_{i}, w_{i}\right) \in D_{M}, i=1,2$;

(H3) $L_{1}:=(q-1) M^{q-2} M_{2}^{q-1}\left(Q_{1} M_{1}+Q_{2}\right)<1$.

Then the mixed boundary value problem (1.1) has a unique solution satisfying the estimation

$$
|y(x)| \leq M_{1} M_{2}^{q-1} M^{q-1}, \quad\left|D_{0+}^{\delta} y(x)\right| \leq M_{2}^{q-1} M^{q-1} \quad \text { for } x \in[0,1] .
$$

Proof First of all, define an operator $A: C[0,1] \rightarrow C[0,1]$ by

$$
\begin{aligned}
(A \phi)(x)= & g\left(x, \int_{0}^{1} G_{1}(x, \tau) \varphi_{q}\left(\int_{0}^{1} G_{2}(\tau, s) \phi(s) d s\right) d \tau,\right. \\
& \left.\varphi_{q}\left(\int_{0}^{1} G_{2}(x, s) \phi(s) d s\right)\right) .
\end{aligned}
$$

From the continuity of $G_{1}(x, \tau), G_{2}(\tau, s)$, and $g(x, y, w)$, it is not hard to see that the operator $A$ is a continuous operator. According to Lemma 2.1, it is easy to get the following conclusion that if the mixed boundary value problem (1.1) has a solution $y(x)$, then $\phi(x)={ }^{c} D_{1-}^{\gamma}\left(\varphi_{p}\left(D_{0+}^{\delta} y(x)\right)\right)$ is the fixed point of the operator $A$. On the contrary, if $\phi(x)$ is a fixed point of the operator $A$, then

$$
y(x)=\int_{0}^{1} G_{1}(x, \tau) \varphi_{q}\left(\int_{0}^{1} G_{2}(\tau, s) \phi(s) d s\right) d \tau
$$

also is the solution of the mixed boundary value problem (1.1).

Next, what we need to prove is that the operator $A$ maps $B[O, M]$ into itself. Given $\phi(x) \in$ $B[O, M]$, by Lemma 2.2 , there is

$$
|y(x)| \leq M_{1} M_{2}^{q-1} M^{q-1}, \quad|w(x)| \leq M_{2}^{q-1} M^{q-1} .
$$

Consequently, for any $x \in[0,1]$, there is $(x, y(x), w(x)) \in D_{M}$. So, from $(H 1)$, we can conclude that

$$
|(A \phi)(x)|=|g(x, y(x), w(x))| \leq M
$$

therefore $(A \phi)(x) \in B[O, M]$. Namely, the operator $A$ is an operator that maps $B[O, M]$ into itself.

Finally, the operator $A: B[O, M] \rightarrow B[O, M]$ is proven to be a contraction mapping. Obviously, $B[O, M]$ is proven to be a complete distance space on account of that $B[O, M]$ is a subspace of $C([0,1],\|\cdot\|)$. From (H2), Lemma 2.2, and (ii) of Lemma 2.3, there is $\left|\int_{0}^{1} G_{2}(\tau, s) \phi(s) d s\right| \leq M_{2} M:=N$ for each $\phi_{1}(x), \phi_{2}(x) \in B[O, M]$ and $1<p \leq 2$, that is, $q \geq 2$, we gain

$$
\begin{aligned}
& \left|\left(A \phi_{2}\right)(x)-\left(A \phi_{1}\right)(x)\right| \\
& \quad=\left|g\left(x, y_{2}(x), w_{2}(x)\right)-g\left(x, y_{1}(x), w_{1}(x)\right)\right| \\
& \quad \leq Q_{1}\left|y_{2}(x)-y_{1}(x)\right|+Q_{2}\left|w_{2}(x)-w_{1}(x)\right|
\end{aligned}
$$




$$
\begin{aligned}
\leq & Q_{1}\left|\int_{0}^{1} G_{1}(x, \tau)\left[\varphi_{q}\left(\int_{0}^{1} G_{2}(\tau, s) \phi_{2}(s) d s\right)-\varphi_{q}\left(\int_{0}^{1} G_{2}(\tau, s) \phi_{1}(s) d s\right)\right] d \tau\right| \\
& +Q_{2}\left|\varphi_{q}\left(\int_{0}^{1} G_{2}(\tau, s) \phi_{2}(s) d s\right)-\varphi_{q}\left(\int_{0}^{1} G_{2}(\tau, s) \phi_{1}(s) d s\right)\right| \\
\leq & Q_{1}(q-1)\left(M_{2} M\right)^{q-2}\left[\int_{0}^{1} G_{1}(x, \tau)\left|\int_{0}^{1} G_{2}(\tau, s)\left(\phi_{2}(s)-\phi_{1}(s)\right) d s\right| d \tau\right] \\
& +Q_{2}(q-1)\left(M_{2} M\right)^{q-2}\left|\int_{0}^{1} G_{2}(\tau, s)\left(\phi_{2}(s)-\phi_{1}(s)\right) d s\right| \\
\leq & Q_{1}(q-1)\left(M_{2} M\right)^{q-2}\left[\int_{0}^{1} G_{1}(x, \tau) \int_{0}^{1} G_{2}(\tau, s) d s d \tau\left\|\phi_{2}-\phi_{1}\right\|\right] \\
& +Q_{2}(q-1)\left(M_{2} M\right)^{q-2}\left[\int_{0}^{1} G_{2}(\tau, s) d s\left\|\phi_{2}-\phi_{1}\right\|\right] \\
\leq & Q_{1}(q-1)\left(M_{2} M\right)^{q-2} M_{1} M_{2}\left\|\phi_{2}-\phi_{1}\right\| \\
& +Q_{2}(q-1)\left(M_{2} M\right)^{q-2} M_{2}\left\|\phi_{2}-\phi_{1}\right\| \\
= & (q-1) M^{q-2} M_{2}^{q-1}\left(Q_{1} M_{1}+Q_{2}\right)\left\|\phi_{2}-\phi_{1}\right\| \\
= & L_{1}\left\|\phi_{2}-\phi_{1}\right\| .
\end{aligned}
$$

Hence,

$$
\left\|\left(A \phi_{2}\right)-\left(A \phi_{1}\right)\right\| \leq L_{1}\left\|\phi_{2}-\phi_{1}\right\|
$$

where $L_{1}<1$ is given by $(H 3)$. Therefore, the operator $A: B[O, M] \rightarrow B[O, M]$ is a contraction mapping. That is to say, there is a unique solution $\mathrm{y}(\mathrm{x})$ of the mixed boundary value problem (1.1) such that

$$
|y(x)| \leq M_{1} M_{2}^{q-1} M^{q-1}, \quad\left|D_{0+}^{\delta} y(x)\right| \leq M_{2}^{q-1} M^{q-1} \quad \text { for } x \in[0,1] .
$$

The proof is completed.

\section{An example}

Example 4.1 Consider the following mixed fractional-order $p$-Laplace differential equation:

$$
\begin{aligned}
& { }^{c} D_{1-}^{\frac{3}{2}}\left(\varphi_{p}\left(D_{0+}^{\frac{3}{2}} y(x)\right)\right)=-2 y^{2}(x) D_{0+}^{\frac{3}{2}} y(x)+3 y(x)-2 D_{0+}^{\frac{3}{2}} y(x)+4 \sin (\pi x), \\
& y(0)=y(1)=D_{0_{+}}^{\frac{3}{2}} y(0)=D_{0_{+}}^{\frac{3}{2}} y(1)=0,
\end{aligned}
$$

where $g(x, y, w)=-2 y^{2} w+3 y-2 w+4 \sin (\pi x)$.

We choose $p=2$, that is, $q=2$. By a simple computation, we obtain that $M_{1}=\frac{1}{8}, M_{2}=\frac{1}{3}$. Then a suitable number $M>0$ is chosen to satisfy all the conditions of Theorem 3.1, and

$$
|y| \leq \frac{M}{24}, \quad|w| \leq \frac{M}{3} .
$$


Obviously, for each $(x, y, w) \in D_{M}$, there is

$$
\begin{aligned}
|g(x, y, w)| & \leq\left|-2 y^{2} w+3 y-2 w+4 \sin (\pi x)\right| \\
& \leq 2\left(\frac{M}{24}\right)^{2}\left(\frac{M}{3}\right)+3\left(\frac{M}{24}\right)+2\left(\frac{M}{3}\right)+4 \sin (\pi x) \\
& \leq M
\end{aligned}
$$

We can easily get that $0<M<13.4164$. Therefore, choose $M=1$, condition $(H 1)$ holds.

Meanwhile, for $(x, y, w) \in D_{M}$,

$$
\begin{aligned}
& \left|g_{y}\right|=|-4 y w+3| \leq 4 \frac{M}{24} \frac{M}{3}+3 \leq 3.5 \\
& \left|g_{w}\right|=\left|-2 y^{2}-2\right| \leq 2\left(\frac{M}{24}\right)^{2}+2 \leq 2.5 .
\end{aligned}
$$

So we choose $Q_{1}=3.5, Q_{2}=2.5$, condition $(H 2)$ holds.

Moreover,

$$
L_{1}=(q-1) M^{q-2} M_{2}^{q-1}\left(Q_{1} M_{1}+Q_{2}\right)=0.979<1,
$$

condition (H3) holds.

So, the above boundary value problem has a unique solution satisfying

$$
|y(x)| \leq \frac{1}{24}, \quad\left|D_{0+}^{\frac{3}{2}} y(x)\right| \leq \frac{1}{3}
$$

\section{Conclusion}

In this article, we have discussed a novel approach which has been put forward by Dang and Bai to prove the existence and uniqueness of solution for a nonlinear multi-point fractional-order $p$-Laplace differential equation, which has at least two different kinds of left and right fractional derivatives. As far as we know, almost nobody explored the work in this area. The advantage of this method is that it is very easy to verify and can be applied to many conditions. Of course, if the boundary condition is changed, the assumptions can be weakened appropriately. Last but not least, the approach mentioned above can be applied to some other boundary value problems, such as conformable fractional order. Furthermore, the boundary conditions can also become integral boundary conditions, and so on.

\section{Acknowledgements}

Not applicable.

Funding

This work is supported by NSFC (11571207), Taishan Scholar project, and SDUST graduate innovation project SDKDYC170343.

Availability of data and materials

Data sharing not applicable to this article as no data sets were generated or analysed during the current study. 
Authors' contributions

All authors read and approved the final manuscript. All authors contributed equally to the writing of this paper.

\section{Publisher's Note}

Springer Nature remains neutral with regard to jurisdictional claims in published maps and institutional affiliations.

Received: 14 September 2020 Accepted: 30 November 2020 Published online: 09 December 2020

\section{References}

1. Ahmad, B., Broom, A., Alsaedi, A., Ntouyas, S.K.: Nonlinear integro-differential equations involving mixed right and left fractional derivatives and integrals with nonlocal boundary data. Mathematics 8(3), 336 (2020)

2. Bai, C.Z.: Existence and uniqueness of solutions for fractional boundary value problems with $p$-Laplacian operator. Adv. Differ. Equ. 2018, 4 (2018)

3. Bai, Z.B., Du, Z.J., Zhang, S.: Iterative method for a class of fourth-order p-Laplacian beam equation. J. Appl. Anal. Comput. 9(4), 1-11 (2019)

4. Dang, Q.A., Dang, Q.L., Quy, N.: A novel efficient method for nonlinear boundary value problems. Numer. Algorithms 76, 427-439 (2017)

5. Dang, Q.A., Nguyen, T.H.: Solving the Dirichlet problem for fully fourth order nonlinear differential equation. Afr. Math. 30, 623-641 (2019)

6. Dang, Q.A., Quy, N.: New fixed point approach for a fully nonlinear fourth order boundary value problem. Bol. Soc. Parana. Mat. 36, 209-223 (2018)

7. Filippucci, R., Pucci, P., Radulescu, V.: Existence and non-existence results for quasilinear elliptic exterior problems with nonlinear boundary conditions. Commun. Partial Differ. Equ. 33, 706-717 (2008)

8. Lakoud, A.G., Khaldi, R., Kılıçman, A.: Existence of solutions for a mixed fractional boundary value problem. Adv. Differ. Equ. 2017, 164 (2017)

9. Liang, S.H., Zhang, J.H.: Positive solutions of boundary value problems of nonlinear fractional differential equation. Nonlinear Anal., Model. Control 71, 5545-5550 (2009)

10. Liu, X.P., Jia, M.: Solvability and numerical simulations for BVPs of fractional coupled systems involving left and right fractional derivatives. Appl. Math. Comput. 353, 230-242 (2019)

11. Liu, X.P., Jia, M., Ge, W.G.: The method of lower and upper solutions for mixed fractional four-point boundary value problem with $p$-Laplacian operator. Appl. Math. Lett. 65, 56-62 (2017)

12. Liu, X.P., Jia, M., Xiang, X.F.: On the solvability of a fractional differential equation model involving the $p$-Laplacian operator. Comput. Math. Appl. 64, 3267-3275 (2012)

13. Reed, M., Simon, B.: IV. Analysis of Operators. Methods of Modern Mathematical Physics. Academic Press, New York (1978)

14. Xu, M.R., Sun, S.R.: Positivity for integral boundary value problems of factional differential equations with two nonlinear terms. J. Appl. Anal. Comput. 59, 271-283 (2019)

\section{Submit your manuscript to a SpringerOpen ${ }^{\circ}$ journal and benefit from:}

- Convenient online submission

- Rigorous peer review

Open access: articles freely available online

- High visibility within the field

- Retaining the copyright to your article 